\title{
Research on farmland crop classification based on UAV multispectral remote sensing images
}

\author{
Dongjian Yang ${ }^{1,2}$, Jing Zhao ${ }^{1,2^{*}}$, Yubin Lan ${ }^{2,3,4,5}$, Yuting Wen ${ }^{1,2}$, Fangjiang Pan ${ }^{1,2}$, \\ Dianlong $\mathrm{Cao}^{1,2}$, Chuanxu $\mathrm{Hu}^{1,2}$, J inkai Guo ${ }^{1,2}$ \\ (1. School of Agricultural Engineering and Food Science, Shandong University of Technology, Zibo 255000, Shandong, China; \\ 2. National International Joint Research Center of Precision Agriculture Aerial Drug Application Technology, Shandong University of \\ Technology, Zibo 255000, China; \\ 3. National Center for International Collaboration Research on Precision Agricultural Aviation Pesticides Spraying Technology/ \\ College of Engineering, South China Agricultural University, Guangzhou 510642, China; \\ 4. Department of Biological and Agricultural Engineering, Texas A\&M University, College Station, Texas, 77843, USA; \\ 5. Texas A\&M AgriLife Research and Extension Center, Beaumont, Texas, 77713, USA)
}

\begin{abstract}
UAV remote sensing technology is used to accurately and efficiently identify crops in farmland, providing technical support for obtaining crop category information in time and realizing precision agriculture. Obtain multi-spectral images of farmland peach trees, corn, weeds and other ground objects with quadrotor equipped with a multi-spectral camera, and use the method of principal component analysis to transform the multi-spectral image, retain the first 2 spectral bands with the most information content, and calculate 16 textures from 7 vegetation indices, 6 reflectances and 2 principal component bands, totaling 29 The item feature is used as the feature parameter of the classification sample. The ReliefF algorithm is used to screen the spectral features and texture features respectively. The 11 features obtained (blue reflectivity, green reflectivity, red edge reflectivity, normalized green band difference vegetation index, enhanced vegetation index, ratio vegetation index, first One principal component correlation, second principal component synergy, information entropy, second moment, correlation). from the screening form the A-group feature data set. All the spectral features extracted from the image form the B-group feature data set and all the extracted texture features form the C-group feature data Set, all 29 features form the D feature data set. SVM support vector machine is used to supervise and classify the 4 groups of feature data sets, and the accuracy of the classification results obtained is evaluated. The accuracy of the supervised classification model trained on the single category feature data sets of B and C is poor, and the SVM supervised classification model trained on the feature data set of the ReliefF algorithm has a better classification effect, with an overall accuracy of $90.09 \%$, and the Kappa coefficient of 0.86 . The SVM model trained on the feature data set D has the best classification effect, with an overall accuracy of $92.01 \%$, and the Kappa coefficient of 0.89 . The ground object classification based on UAV multi-spectral images is efficient and feasible, which provides a reference for timely acquisition of crop planting structure in farmland.
\end{abstract}

Keywords: UAV, Ground object identification, Multispectral remote sensing, ReliefF algorithm

DOI: $10.33440 /$ j.ijpaa.20210401.153

Citation: Yang D J, Zhao J, Lan Y B, Wen Y T, Pan F J, Cao D L, Hu C X, Guo J K. Research on farmland crop classification based on UAV multispectral remote sensing images. Int J Precis Agric Aviat, 2021; 4(1): $29-35$.

\section{Introduction}

The core of precision agriculture ${ }^{[1-5]}$ is to use information technology to accurately grasp the information of field crops in time to achieve precise irrigation, spraying and fertilization in each

\section{Received date: 2021-02-18 Accepted date: 2021-03-29}

Biographies: Dongjian Yang, Postgraduate student, research interests: remote sensing Mechanical design and computer programming. Email: 694415363@qq.com; Yubin Lan, PhD, professor, Director, research interests: precision agricultural aviation application, Email: ylan@scau.edu.cn; Yuting Wen, Postgraduate student, research interests: remote sensing, Email: 1256596123@qq.com; Fangjiang Pan, Postgraduate student, research interests: remote sensing, Email: 181831794@139.com; Dianlong Cao, Postgraduate student, research interests: remote sensing, Email: 1070414967@qq.com; Chuanxu Hu, Postgraduate student, research interests: Unmanned aerial remote sensing, Email: 416458843@qq.com; Jinkai Guo, Postgraduate student research interests: Unmanned aerial remote sensing, Email: 347060630@qq.com.

* Corresponding author: Jing Zhao, Associate professor, research interests: remote sensing, Mailing Address: Shandong University of Technology West Campus. Email: zbceozj@163.com. area. Efficient and accurate acquisition and identification of field features are the basis for precision agriculture. UAV remote sensing technology can quickly acquire crop image data in large areas, which provides the possibility for efficient extraction of field crop categories. The identification of typical crops is a hot topic in the current agricultural research. Using drone remote sensing technology to quickly and accurately obtain the types of farmland crops is of great significance to the realization of precision agriculture. Currently, there is extensive research in the field of satellite remote sensing for farmland feature recognition. Bai $\mathrm{Xue}^{[6]}$ and others used Landsat 8 and Gaofen No. 1 remote sensing images to investigate crop planting information and select different supervision classification methods. The main crops in shawan county have been identified and classified, and satisfied experimental results is achieved. Wanga ${ }^{[7]}$ et al. used multi-temporal Landsat satellite images to perform Fourier transform processing and extract multiple vegetation indices from them, and selected feature values extracted from different crops for optimal selection, and used random forest and unsupervised 
Gaussian mixture models for crop classification, Liu $\mathrm{Jia}^{[8]}$ et al. used the feature extraction process of the NDVI time series curve of HJ-1A/B images to obtain the obvious characteristics of bulk crops, which confirmed the feasibility of crops classification for $\mathrm{HJ}$ satellite time series images. Although satellite remote sensing technology has become mature in ground feature recognition, it has problems such as susceptibility to weather conditions, poor effectiveness, and low temporal and spatial resolution. At the same time, it is often difficult to meet the extraction of ground feature recognition information at the field scale. It is difficult to achieve the purpose of precise fertilization and medication.

UAV remote sensing technology has the advantages of high feasibility, strong real-time performance, low cost, convenience and high efficiency. It is widely used in the acquisition of remote sensing data in small areas. Compared with satellite remote sensing data, UAV remote sensing data has higher time resolution, and is more appropriate to extract. the spectrum, texture and geometric characteristics of various ground objects, making it possible to obtain crop information efficiently and accurately. At present, in the application of ground feature recognition and classification, the UAV platform is usually equipped with a digital camera to capture the farmland planting information. Mitch Bryson ${ }^{[9]}$ proposed a method of using low-altitude drones to collect visible light data. Based on visible light images to extract the color and texture features of vegetation, which is used for large-scale terrain classification with good results. Gnadinger ${ }^{[10]}$ and others used drone remote sensing technology to obtain visible light images of field crop canopy and analyze the image data, and realized the accurate recognition of corn seedlings through the threshold method, which provided a new method for fast and accurate farmland crop classification. Dai Jianguo ${ }^{[11]}$ obtained crop visible light images through drones, performed color space conversion and texture filtering processing on the images, filtered and trained the extracted features to obtain a classification model, combined with manual classification results to achieve accurate classification of crops. Han Wenting ${ }^{[12]}$ and others used UAV remote sensing technology to obtain visible light images of the field, extract multiple texture features of the five types of ground objects in the image, and achieve the extraction of corn planting information by filtering single classification features and combining hierarchical classification features. The above studies are based on UAV remote sensing technology to collect visible light image data to classify and recognize features. The visible light data only contains three bands of RGB, which contains less spectral information and the process of extracting corresponding features of different features is relatively complicated. Compared with visible light, Multispectral sensors contain more spectral information, and have more advantages in identifying the types of ground objects. Dai Jianguo ${ }^{[13]}$ and others used UAV multi-spectral images to extract the spectral reflectance information of normal cotton and Lodging cotton, and established the best model of cotton Lodging disaster monitoring. Cui Meina ${ }^{[14]}$ and others used unmanned aerial vehicle remote sensing technology to obtain multispectral images of the test area, constructed 20 spectral index characteristic factors for the target area, and successfully constructed a logistic regression monitoring model for the identification of cotton field mites. Dai Jianguo ${ }^{[15]}$ and others used unmanned aerial vehicles to obtain visible light images of the test area, extracted texture features and low-pass filtering features of various objects in the test area, and used ReliefF-Pearson algorithm feature selection to perform data dimensionality reduction to obtain the best feature set. Five supervised classification models were used to classify and identify the crops in the verification area, and it was found that the SVM was used to classify the best results. Many scholars use UAV multi-spectral images to classify farmland features. There are disadvantages such as complex methods, low efficiency, and high cost. UAV visible light remote sensing data has a small amount of image spectral information, and the vegetation index that can be constructed is limited, causing problems such as low classification accuracy. To solve this problem, UAV multi-spectral remote sensing data is used, which has more spectral bands, so it can be constructed a variety of vegetation indices are used for the information extraction of farmland crop characteristics, and at the same time, the feature screening method is used to reduce the dimension of the feature data, which improves the recognition efficiency and guarantees the classification accuracy of the farmland crops. This paper uses drones equipped with multi-spectral cameras to obtain high-resolution crop remote sensing image data containing multi-band spectral information, and studies the rapid and accurate acquisition of farmland based on drone multi-spectral remote sensing images. The method of crop classification provides a reference for timely and efficient acquisition of farmland feature categories. UAV remote sensing technology is used to collect multi-spectral images of farmland crops. By extracting the vegetation index, spectral reflectance and texture characteristics of different ground objects, the peach trees, corn, weeds and other ground objects in the study area are analyzed. The planting structure information is extracted and accuracy verified. The SVM is used to supervise and classify the feature data set, and the pixel-level semantic recognition of a variety of crops in the test area is provided, which provides a method for quickly identifying different types of ground objects in the field, to provide a reference for UAV multi-spectral remote sensing in farmland feature type identification.

\section{Materials and Methods}

\subsection{Study area and data acquisition}

The test site is a hilly farmland in Xiaoli Village $\left(36^{\circ} 21^{\prime} 4.10^{\prime \prime} \mathrm{N}, 118^{\circ} 05^{\prime} 44.62^{\prime \prime} \mathrm{E}\right)$, Boshan District, Zibo City, Shandong Province. The terrain is undulating, with various crop types and complex planting structures. This area belongs to the temperate monsoon climate. The image data was collected on June 25, 2020, and the collection period was 11:00-12:30. The drone is selected by Shenzhen DJI Technology Co., Ltd. Jingwei M210V1 quad-rotor drone with a flight time of 20 minutes. The machine is equipped with an MS600Pro multi-spectral camera to obtain image data. The sensor captures 6 channels, including blue, green, red, red edges, near-infrared $(840 \mathrm{~nm})$ and near infrared $(940 \mathrm{~nm})$. The image resolution is $1280 \times 960$, and the captured image is stored in the SD card. On the day of data collection, the weather was clear and Southeast wind 1, which met the requirements of drone flight. The experimental design of the UAV has a flying height of $80 \mathrm{~m}$, a flying speed of $4 \mathrm{~m} / \mathrm{s}$, a heading overlap of $85 \%$, and a side overlap of $85 \%$. Images are collected in a vertical manner, and a total of 2628 images of 6 single-channel images are obtained.

First, the YusenseRef software was used to perform radiometric correction on all the images obtained in the test area, and then the pix4Dmapper software was used to perform geometric correction and image stitching on all single images after radiometric correction. According to the POS data collected on 
the fly, find the splicing points of the pictures, and stitch the pictures, Calculate the original image to generate a point cloud mode. and finally add texture data to obtain 6 single-band images, including blue, green, red, red edges, near-infrared $(840 \mathrm{~nm})$, near-infrared $(940 \mathrm{~nm})$ bands. Through the Layer Stacking function of ENVI software, 6 single-band images are fused into a multi-spectral image, and three RGB bands are selected for display after fusion, as shown in Figure 1.

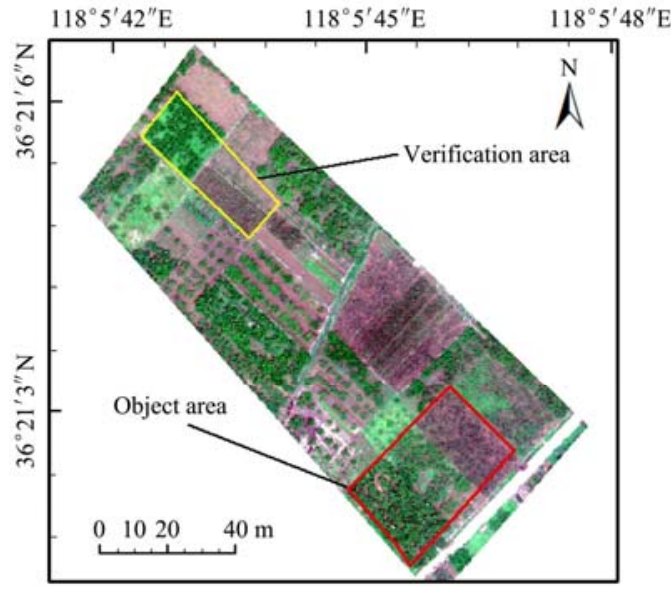

Figure 1 Experimental region

\subsection{Research approach}

This experiment takes the multi-spectral image collected from farmland in the hilly and mountainous areas as the research object. The principal component analysis algorithm (PCA) is used to reduce the dimensionality of the multi-spectral image to extract peach trees, corn, weeds and other (naked trees) in the region of interest. The reflectance, vegetation index and texture features of ground, shadow, etc. are used as classification features. The extracted features are classified and selected to obtain 4 feature data sets, and SVM (support vector machine) is used to supervise and classify the 4 feature data sets. After performing 10-fold cross-validation on the resulting classification model, the classification accuracy is compared, and the classification model is used to identify the type of features in the verification area, and finally the confusion matrix accuracy verification is performed on the classification results obtained from each feature set. The technical route is shown in Figure 2.

\subsection{Data Processing}

This article selects 7 commonly used vegetation indices ${ }^{[16-23]}$. SAVI, OSAVI, GNDVI, NDVI, DVI, RVI, and EVI, and performs band calculations through the Band Math function of the ENVI software to obtain 7 vegetation index grayscale images. The Layer stacking function in ENVI fuses the obtained 7 planting index grayscale image with the original 6-band spectral reflectance image to obtain an image containing 7 vegetation index and 6 spectral reflectance information, which constitutes a classified spectral feature set.

Each band of the multispectral image contains a large amount of information, and there is information redundancy between the bands. In order to reduce the correlation between the bands and reduce the information redundancy phenomenon, this experiment uses the principal component analysis method ${ }^{[24-26]}$ (principal component analysis, PCA) to perform data dimensionality reduction on multispectral data.

The PCA transformed multi-spectral image has 6 principal components, and the information content is $98.25 \%, 1.52 \%, 0.17 \%$, $0.04 \%, 0.017 \%, 0.003 \%$, respectively. Because the accumulated information of the first two principal components accounted for $99.77 \%$ of the total data information, this paper selects only the first two principal components that contain the most information, and discards the remaining four principal components.

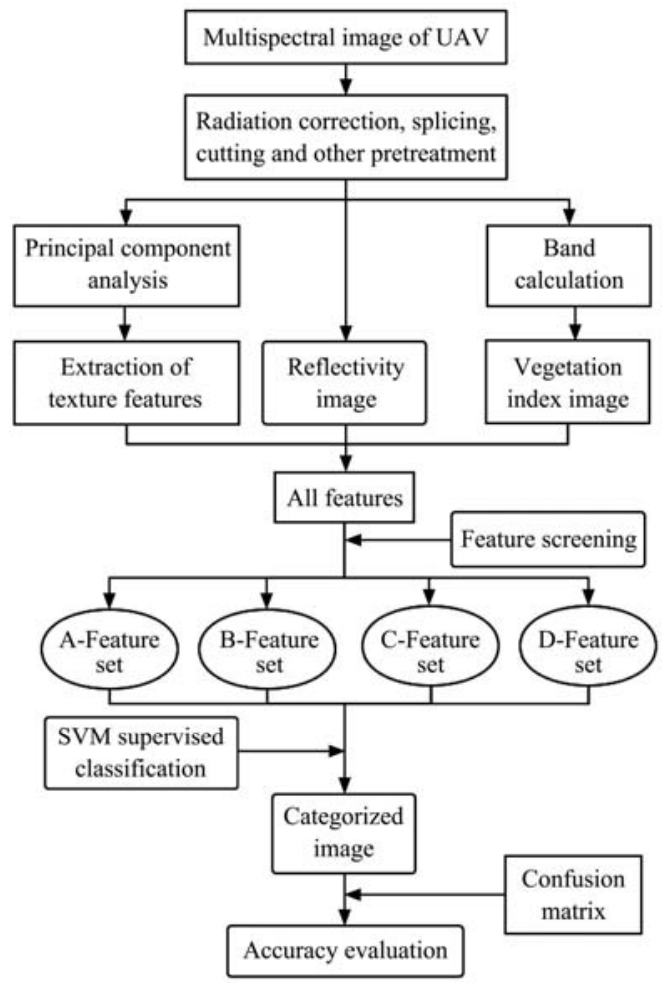

Figure 2 Technology roadmap

\subsection{Feature Processing}

\subsubsection{Feature Extraction}

The phenomenon of different spectra and foreign objects of the same spectrum in the classification of multi-spectral image features is an important reason for the reduction of classification accuracy. In order to suppress the occurrence of this phenomenon, reduce misclassification and omission, and improve classification accuracy, the following two methods are usually used: one is to further process the spectral information to improve this situation, and the other is to introduce new classification features and integrate multiple features to accurately identify ground objects. As an image feature, texture can not only consider the grayscale difference of the pixel itself, but also highlight the difference in the relationship between each pixel. Therefore, it is selected to extract the texture features of peach trees, corn, weeds and other ground objects in the multispectral image. At present, the most used texture analysis method is the gray-level co-occurrence matrix $\operatorname{method}^{[27-30]}$. This paper chooses to calculate 8 texture feature parameters including: Mean, Variance, Homogeneity, Contrast, Dissimilarity, Entropy, Angular Second Moment, Correlation.

The gray-level co-occurrence matrix describes the probability that a pair of pixels separated by $d$ pixels in the direction $\theta$ have gray levels $i$ and $j$, respectively, and its elements can be denoted as $P(i, j \mid d, \theta)$. When $\theta$ and $d$ When selected, it is abbreviated as $P i j$, and its order is determined by the number of gray-scale quantization layers of the image. A set of parameters can be calculated from the gray-level co-occurrence matrix and used to quantitatively describe the texture characteristics of the image. For example, the contrast is gray-scale A measure of uniformity of distribution. The more uneven the distribution, the greater the difference in $P i j$ and the greater the contrast; correlation can be used to describe the degree of similarity between row elements or 
column elements in the Pij matrix. It is a measure of the linear relationship of gray levels. Its expression formula is as follows: (1).

$P(i, j \mid \mathrm{d}, \theta)=\#\{(x, y) \mid f(x, y)=i, f(x+d x, y+d y)=j ;$

$$
x, y=0,1,2, \ldots, N-1\}
$$
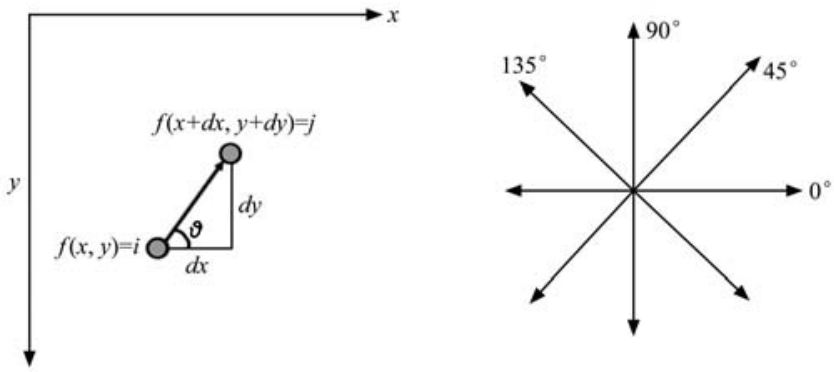

Figure 3 Gray Level Co-occurrence Matrix

In the horizontal direction, which is the 0 direction, there are a total of 4 of $0-0$; a total of 2 of $0-1$.
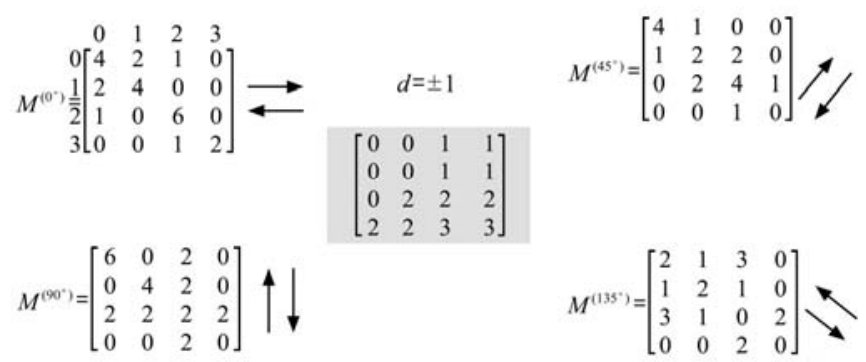

\section{Figure 4 Calculation}

The gray-level co-occurrence matrix provides information on the image gray direction, interval, amplitude of change, and speed, but it cannot directly provide the characteristics of different textures. Therefore, it is necessary to extract statistics used to quantitatively describe texture features on the basis of the gray-level co-occurrence matrix Attributes.Select eight commonly used feature statistics used to extract texture information from remote sensing images as shown in the following Table 1.

Table 1 Texture feature

\begin{tabular}{cl}
\hline Name & Formula \\
\hline Variance & $\frac{1}{M N} \sum_{i=0}^{M-1} \sum_{j=0}^{N-1} f(i, j, d, \theta)$ \\
Homogeneity & $\sum_{i=0}^{M-1} \sum_{j=0}^{N-1}(i-\mu)^{2} f(i, j, d, \theta)$ \\
Contrast & $\sum_{i=0}^{M-1} \sum_{j=0}^{N-1} \frac{f(i, j, d, \theta)}{1+(i-j)^{2}}$ \\
Dissimilarity & $\sum_{i=0}^{M-1} \sum_{j=0}^{N-1}(i-j)^{2} f(i, j, d, \theta)$ \\
Entropy & $\sum_{i=0}^{M-1} \sum_{j=0}^{N-1}|i-j| f(i, j, d, \theta)$ \\
Angular Second Moment & $-\sum_{i=0}^{M-1} \sum_{j=0}^{M-1} f(i, j) \lg f(i, j, d, \theta)$ \\
& $\sum_{i=0}^{M-1} \sum_{j=0}^{N-1} f(i, j, d, \theta)^{2}$ \\
Correlation & $\sum_{i=0}^{M-1} \sum_{j=0}^{N-1} \frac{(i-\mu)(j-\mu) f(i, j, d, \theta)^{2}}{\delta^{2}}$ \\
\hline
\end{tabular}

Through the Co-occurrence-Measures function of ENVI software, the second-order probability statistical filter is used to calculate the first two principal component bands after PCA dimensionality reduction, and the texture features of each image are extracted to form a feature set containing 16 texture features. The filter window size is set to $7 \times 7$, the variation of the spatial correlation matrix $\mathrm{X}$ and $\mathrm{Y}$ are both set to 1 , and the gray level is set to 32 .

\subsubsection{Feature set selection}

In order to improve model operation efficiency, reduce data set dimensions and eliminate information redundancy, this paper chooses to use ReliefF algorithm to reduce the dimensionality of the original data set. The purpose of feature selection is to reduce the dimensionality, reduce the difficulty of learning tasks and improve the efficiency of the model. The common feature selection methods mainly include four types: filtering, wrapping, filtering and wrapping combined algorithms, and embedded ${ }^{[24,28]}$.

The ReliefF algorithm is a typical filter selection method ${ }^{[31-34]}$. The algorithm is simple and has high operating efficiency, so it is widely used. When the ReliefF algorithm deals with multiple types of problems, it randomly takes a sample $\mathrm{R}$ from the training sample set each time. And then find the $\mathrm{k}$ nearest neighbor samples (near Hits) of R from the sample set of the same kind as R, find $k$ nearest neighbor samples (near Misses) from the sample set of different classes of each $\mathrm{R}$, and then update the weight of each feature to make it suitable for the feature screening and dimensionality reduction in this paper.

There are many methods to reduce the dimensionality of data and eliminate information redundancy. At present, feature selection algorithms are widely used to weight all the extracted feature data and select the best processing. This paper proposes a method to reduce the dimensionality of different feature attributes to extract feature parameters, The ReliefF algorithm is used to screen texture features and spectral features respectively, and the screening results are used to supervise the training of the classification model. The ReliefF feature selection algorithm is implemented by Python software programming, which calculates and sorts the classification weights of 16 texture features and 13 spectral features respectively. The spectral features are all features with a weight value greater than 0.001 , and the texture features are all features with a weight greater than 0.01. A total of 11 features constitute the feature data set of Group A, which are: (blue reflectivity, green reflectivity, red edge reflectivity, normalized green band difference vegetation index, enhanced vegetation index, ratio vegetation index, first One principal component correlation, second principal component synergy, information entropy, second moment, correlation).

Select 16 texture feature parameters to form group B data, 13 spectral feature parameters to form group $\mathrm{C}$ data, and all 29 feature parameters to form group D data.

\subsection{Supervise the construction of classification models}

The test is divided into the target area and the verification area. Figure 5 shows the RGB images of the verification area and the target area respectively. The verification area has been investigated on the type of features on the spot, and the features in the verification area are divided into the area of interest as the ground truth through manual selection. The region of interest is selected with a random point as the center, and the feature average within the range of $3 \times 3$ pixels is used as the ground truth. Sample feature data to ensure that each region of interest contains 9 pixels.

The ENVI software ROI tool was used to select sample points in the target area and the verification area. The ground objects in the target area are peach trees, corn, weeds, and other ground objects (bare land and shadows, etc.), and the ground objects in the verification area are corn, weeds, etc., Peach trees, and other features. After collecting images on the day of the test, a field 
survey was conducted to determine the actual features and verify them as true values. Manually classify various features in the image and select the region of interest (ROI). Each ROI represents a sample of data. In the target area, the interest areas of various features was selected: 140 peach trees, 60 weeds, 100 corn, and 120 other features. The verification area selects the interest areas of various features: 60 peach trees, 35 weeds, 30 corn and 60 other ground features. The statistical values of texture, vegetation index and reflectance extracted from each region of interest of peach trees, corn, weeds, and other types of ground objects are used as sample features.

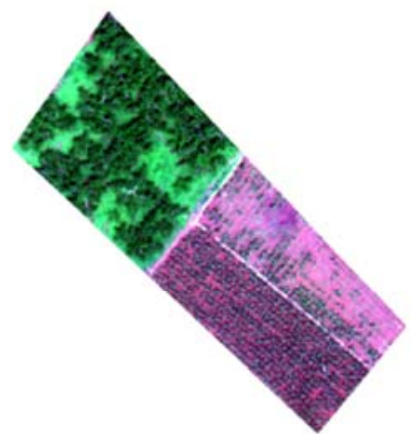

a. Verification area

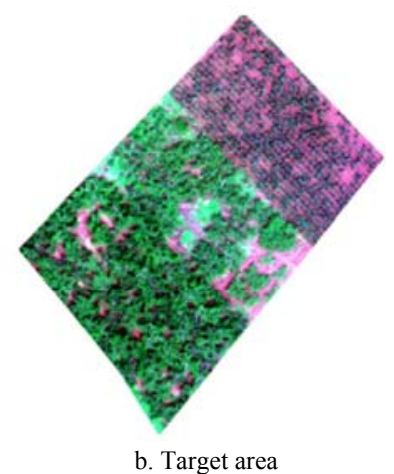

b. Target area
Figure 5 Verification area and target area

The feature data in all data sets in the target area are randomly divided into training set and test set at a ratio of $3: 1$. The training set is used for the training of the supervised model, and the test set is used for the accuracy verification of the feature data set and the model selection. The SVM is used to supervise and classify each feature set. All data are normalized, and the classification model is trained using 10-fold cross-validation. All processing is implemented by Python programming.

\section{Experimental results and analysis}

\subsection{Precision appraising}

Use SVM to supervise and classify 4 sets of feature data sets to obtain classification models with different classification accuracy. This article uses Accuracy, Precision, Recall and $F_{1}$ values to pair A, B, C, D. 4 types of feature sets are trained for classification results for accuracy evaluation. The calculation methods of the four evaluation indicators ${ }^{[15]}$ are as follows:

$$
\begin{gathered}
A C C=\frac{T P+T N}{T P+T N+F P+F N} \\
P=\frac{T P}{T P+F P} \\
R=\frac{T P}{T P+F N} \\
F_{1}=\frac{2 P R}{P+R}
\end{gathered}
$$

In the formula: TP, TN, FP, FN are true cases, true negative cases, false positive cases, and false negative cases respectively. The accuracy rate is the proportion of the number of correct predictions to the total number of samples, and the accuracy rate represents the cases classified as positive cases Actual is the proportion of real cases, and the recall rate is the proportion of all cases that are actually positive cases are predicted to be positive cases, which is equivalent to Sensitive. The classification accuracy evaluation results obtained by using SVM to supervise and classify the 4 types of feature data sets are shown in the table. Shown in 2.
Table 2 Evaluation and comparison of classification accuracy of four feature sets

\begin{tabular}{cccccc}
\hline \multirow{2}{*}{$\begin{array}{c}\text { Feature } \\
\text { set }\end{array}$} & $\begin{array}{c}\text { Accuracy of } \\
\text { training set }\end{array}$ & \multicolumn{5}{c}{ Test set } \\
\cline { 3 - 6 } & & Accuracy/\% & Precision/\% & Recall/\% & $\mathrm{F}_{1}$ \\
\hline A & 91.324 & 88.774 & 90.428 & 90.327 & 90.377 \\
B & 47.273 & 53.860 & 43.590 & 85.00 & 57.627 \\
C & 90.864 & 87.734 & 87.652 & 87.413 & 87.532 \\
D & 93.436 & 90.649 & 92.631 & 92.574 & 92.602 \\
\hline
\end{tabular}

As shown in Table 2, the classification accuracy of the classification model derived from the $\mathrm{D}$ feature data set is the highest, and the accuracy of the test set can reach $90.649 \%$. The classification model derived from the A feature data set is better than the $\mathrm{C}$ feature data set. The classification model obtained from the training of the B feature data set has the worst accuracy. The results show that the full feature data set has the best effect on the construction of the classification model, and the accuracy is the highest. The classification model obtained from the spectral feature data set training has the best effect on the test set. The accuracy rate can reach $87.734 \%$. Using the ReliefF algorithm to filter texture and spectral features separately, the feature data set obtained by dimensionality reduction is used to construct the classification model. The effect of the classifier is better than the two single feature data sets, and the accuracy of the test set is up to $88.774 \%$, the accuracy rate of the classification model constructed by the texture feature set is the worst.

\subsection{Verify the comparison and analysis of the effect of region classification}

SVM support vector machine is used to supervise and classify 4 sets of feature data sets, and the training classification model is used to identify the features in the verification area image, and the features in the verification area are verified through the classification of the features obtained from the field survey and manual selection. The sample of interest is selected as the ground truth value for verification. The classification image of the verification area is shown in Figure 6, and the confusion matrix of the classification result is shown in Table 3 .

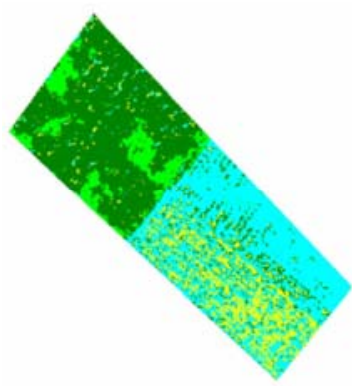

c. ReliefF feature set

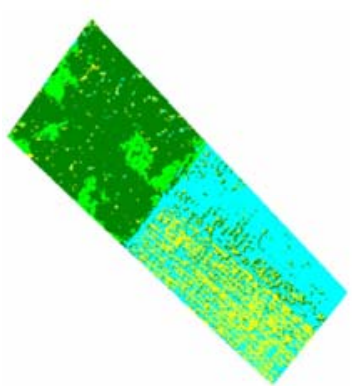

e. Spectral feature set

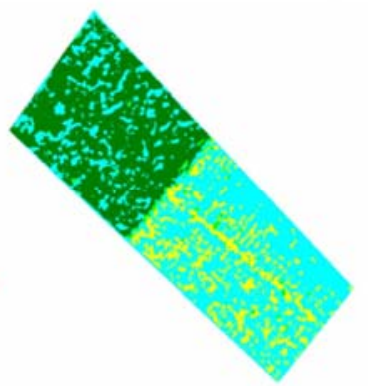

d. Texture feature set

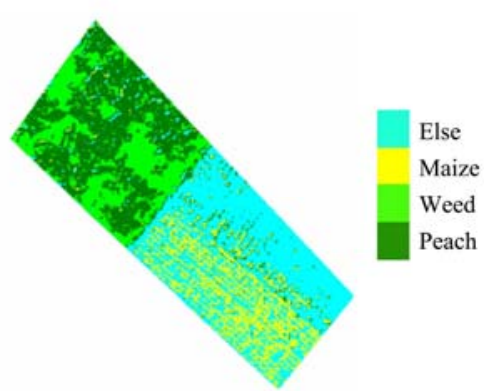

f. Whole feature set
Figure 6 Verification on the classification effect of different feature sets in the area 
Table 3 The result of verification zone classification confuses the matrix

\begin{tabular}{cccccccc}
\hline $\begin{array}{c}\text { Feature } \\
\text { set }\end{array}$ & $\begin{array}{c}\text { Relief } \\
\text { objects }\end{array}$ & Else & Peach & Mazie & Weed & Kappa $\begin{array}{c}\text { Overall } \\
\text { accuracy }\end{array}$ \\
\hline \multirow{2}{*}{ A } & Else & 98.33 & 0.19 & 0.37 & 0 & & \\
& Peach & 1.3 & 96.85 & 31.48 & 16.51 & 0.86 & 90.09 \\
& Mazie & 0.37 & 1.48 & 67.78 & 0 & & \\
& Weed & 0 & 1.48 & 0.37 & 83.49 & & \\
\hline \multirow{2}{*}{ B } & Else & 91.30 & 19.81 & 52.59 & 21.27 & & \\
& Peach & 0.19 & 80.19 & 0 & 78.73 & 0.42 & 59.64 \\
& Mazie & 8.33 & 0 & 24.81 & 0 & & \\
& Weed & 0.19 & 0 & 22.59 & 0 & & \\
& Else & 96.3 & 0 & 0.37 & 0.95 & & \\
C & Peach & 2.04 & 94.63 & 27.41 & 31.75 & 0.81 & 86.12 \\
& Mazie & 8.33 & 4.63 & 70.74 & 0 & & \\
& Weed & 0.19 & 0.74 & 1.48 & 67.3 & & \\
\hline \multirow{2}{*}{ D } & Else & 100 & 0.19 & 0 & 2.22 & & \\
& Peach & 0 & 80.37 & 6.67 & 0.63 & 0.89 & 92.01 \\
& Mazie & 0 & 0.19 & 93.33 & 0 & & \\
& Weed & 0 & 19.26 & 0 & 97.14 & & \\
\hline
\end{tabular}

The results show that the classification accuracy of feature data set $\mathrm{D}$ is the highest, the overall accuracy can reach $92.01 \%$, and the Kappa coefficient is 0.89 . The overall accuracy of the classification results obtained from the A feature data set is $90.09 \%$, and the Kappa coefficient is 0.86 . The accuracy of the classification result obtained from the $\mathrm{C}$ feature set is lower than the accuracy of the classification result of the A group, and the classification result of the B feature set is the worst. From the classification result, the texture feature cannot effectively distinguish the two types of peach and weed features, and there is a misclassification between the features and the phenomenon of missing points, resulting in a decrease in classification accuracy.

The performance of using a single category feature is poor. The overall accuracy of the classification results obtained from the $\mathrm{B}$ and $\mathrm{C}$ feature sets are lower than the $\mathrm{A}$ and $\mathrm{D}$ feature sets. The $\mathrm{D}$ data set contains all the feature data and the classification results have the highest accuracy, the data set of group A is the feature set obtained by reducing the dimensionality of the spectral features and texture features by the ReliefF algorithm. It contains 11 features, which is 18 fewer features than the full feature set. However, this method takes into account the spectral and texture features in a balanced manner. It ensures that the two major features are reasonably used for the classification and recognition of features. Although the overall accuracy of the classification result of the A feature data set is lower than that of the $\mathrm{D}$ feature data set, the accuracy is reduced to a small extent, and the error is within an acceptable range. The dimensionality reduction of the data set can improve the computational efficiency of the model, screen the features that have high correlation and get rid of features less contributions.

\section{Discussion}

Satellite remote sensing data is mostly used in the research of ground feature classification. Due to its high cost, large impact by weather, low resolution, etc., it is not suitable for the classification research of small area farmland. UAV remote sensing is widely used in in the classification research of farmland for its advantages of feasibility, low cost, and high spatial resolution. This paper applied UAV remote sensing technology to obtain farmland multispectral images through feature extraction to construct feature data sets, and SVM support vector machines are used to analyze different feature sets. Supervised classification is carried out, and a better method for classification of farmland multispectral image features is obtained. This research provides a reference for quickly obtaining farmland planting structure. However, there are many types of farmland crops and complex planting structures. The collected images are affected by time and weather. The green vegetation has shadows. At the same time, the difference in spectral reflectance of different green vegetation in the test area is small, and there is a phenomenon that the same spectrum corresponds to different crops. The texture characteristics of maize and weeds in the seedling stage are less different. These have caused the reduction of ground feature recognition accuracy. For peach trees and maize and weeds, there are omissions and misclassifications, and the DSM elevation information may be added in the later period. The classification accuracy of the ground features, and the use of multi-temporal data to classify the ground features can be considered, which will be explored in the next experiment.

\section{Conclusion}

Through the ReliefF algorithm and the combination of different feature attributes, two feature selection methods are used to filter the crop texture features and spectral features of the multi-spectral image after the dimensionality reduction of the principal component analysis, and the four sets of feature data are monitored through the support vector machine classification model. Set classification, compare the classification accuracy and classification results, obtaining the optimal classification feature data set, and realizing the efficient and accurate classification of different crops in the farmland. The results show that:

Use the ReliefF algorithm to screen the extracted spectral and texture features, and use SVM to supervise and classify the feature data set, verifying that the overall classification accuracy is $90.09 \%$, and the Kappa coefficient is 0.86 .The classification model obtained by fusing the full feature set of multiple features including all texture and spectral feature information has the best classification effect. The classification accuracy of the test set can reach $90.649 \%$, the overall classification accuracy of verification is $92.01 \%$, and the Kappa coefficient is 0.89 . Comparing the classification results obtained from different feature sets, the group $\mathrm{D}$ with full feature data set has the best classification effect, the B and $\mathrm{C}$ group feature data sets have poor classification accuracy, and the A group feature data set has better classification results, and the A group feature data set has better classification results, with better efficiency.

\section{Acknowledgments}

This study was supported by Top Talents Program for One Case One Discussion of Shandong Province and the Development Special Funds on Science and Technology to Guide Local by the Central Government"Research and Development on Techonology and Equipment of Precision Agriculture Aviation".

\section{[References]}

[1] Zhou Z Y, Ming R, Zang Y, He X G, Luo X W, Lan Y B. Development status and countermeasures of agricultural aviation in China. Transactions of the Chinese Society of Agricultural Engineering, 2017; 33(20): 1-13. (in Chinese) doi: 10.11975/j.issn.1002-6819.2017.20.001.

[2] Lan Y B. Current status and future prospects of precision agricultural 
aviation technology. Agricultural Engineering Technology, 2017; 37(30): 27-30. (in Chinese) doi: 10.16815/j.cnki.11-5436/s.2017.30.003.

[3] He D J, He Y, Li M Z, Hong T S, Wang C H, Song S, Liu Y G. Research progress of information science-related problems in precision agriculture. Bulletin of National Natural Science Foundation of China, 2011; 25(1): 10-16. (in Chinese) doi: 10.16262/j.cnki.1000-8217.2011.01.006

[4] Lan Y B, Wang G B. China's plant protection drone industry development overview and development prospects. Agricultural Engineering Technology, 2018; 38(9): 17-27. (in Chinese) doi: 10.16815/j.cnki.11-5436/s.2018.09.004

[5] Shi Z, Liang Z Z, Yang Y Y, Guo Y. Status and prospect of agricultural remote sensing. Transactions of the Chinese Society for Agricultural Machinery, 2015; 46(2): 247-260. (in Chinese)

[6] Bai X, identification of crop species in shawan county based on Landsat8 and gaofen-1 images, shandong agricultural sciences, 2020; 52(2): 156-162. (in Chinese) doi: 10.14083/j.issn.1001-4942.2020.02.029.

[7] wanga S, Azzari G, Lobell D B. Crop type mapping without field-level labels; random forest transfer and unsupervised clusteringtechniques. Remote Sensing of Environment, 2019; 222: 303-317. doi: 10.1016/ j.rse.2018.12.026

[8] Liu J, Wang L L, Yang F, etal. remote sensing estimation of crop planting area based on HJ time-series images, Transactions of the Chinese Society of Agricultural Engineer- ing, 2015; 31(3): 199-206. (in Chinese)

[9] Mitch, Bryson, Alistair, et al. Airbome vision - based mappingand classification of large farmland environments. Journal ofField Robotics, 2010; 27(5): 632-655. doi: 10.1002/rob.20343

[10] Friederike G, Urs S. Digital Counts of Maize Plants by Un-manned Aerial Vehicles (UAVs). Remote Sensing, 2017; 9(6): $544 . \quad$ doi: 10.3390/rs9060544

[11] Dai J G, Zhang G S, Guo P, et al. Classification Method of Main Crops in Northern Xinjiang Based on UAV Remote Sensing Visible Image Ding.Transactions of the Chinese Society of Agricultural Engineering, 2018; 34(18): 122-129. (in Chinese)

[12] Han W T, Li G, Yuan M M, et al. Research on Extraction Method of Corn Planting Information Based on UAV Remote Sensing Technology Ding.Transactions of the Chinese Society for Agricultural Machinery, 2017; 48(1): 139-147. (in Chinese)

[13] Dai J G, Zhang G S, Guo P, et al. Informationextraction of cotton lodging based on multi - spectral image from UAV remote sensing. Transactions of the Chinese Society ofAgricultural Engineering, 2019; 35(2): 63-70. (in Chinese)

[14] Cui M N, Dai J G, Wang S H, et al. Research on 1-dentification Method of Mite Infection Cotton Based on of UAV Multi-Spectral Image. Xinjiang Agricultural Sciences, 2018; 55(8): 1457-1466. (in Chinese)

[15] DAI J G, Zhang G S, Guo P, et al. Classification method of main crops in northern Xinjiang based on UAV remote sensing visible image. Transactions of the Chinese Society of Agricultural Engineering, 2018; 34(18): 122-129. (in Chinese)

[16] Liu C, Yang G J, Li Z H, Tang F Q, Wang J W, Zhang C L, Zhang L Y. Biomass estimation in winter wheat by UAV spectral information and texture information fusion. Scientia Agricultura Sinica, 2018; 51(16): 3060-3073. (in Chinese)

[17] Rouse J W, Haas R W, Schell J A, Deering D W, Harlan J C. Monitoring the vernal advancement and retrogradation (Greenwave effect) of natural vegetation Final Rep. RSC 1978-4. Remote Sensing Center, Texas
A\&M University, College Station, 1974.

[18] Jordan C F. Derivation of leaf-area index from quality of light on the forest floor. Ecology, 1969; 50: 663-666. doi: 10.2307/1936256.

[19] Pearson R L, Miller L D. Remote mapping of standing crop biomass for estimation of the productivity of the short-grass prairie. Proceedings of the 8th International Symposium on Remote Sensing of Environment. Environmental Research Institute of Michigan. Ann Arbor, MI, USA, 1972 1357-1381. doi: 10.1177/002076409904500102.

[20] Huete A R. A soil-adjusted vegetation index (SAVI). Remote Sensing of Environment, 1988; 25: 295-309. doi: 10.1016/0034-4257(88)90106-X.

[21] Rondeaux G, Steven M, Baret F. Optimization of soiladjusted vegetation indices. Remote Sensing of Environment, 1996; 55: 95-107.

[22] Gitelson A A, Kaufman Y J, Merzlyak M N. Use of a green channel in remote sensing of global vegetation from EOSMODIS. Remote Sensing of Environment, 1996; 58: 289-298. doi: 10.1016/S0034-4257(96) 00072-7.

[23] Louargant M, Villette S, Jones G, Vigneau N, Paoli J, Gee C. Weed detection by UAV: Simulation of the impact of spectral mixing in multispectral images. Precision Agriculture, 2017; 18: 932-951. doi: 10.1007/s11119-017-9528-3.

[24] Zhou Z H. Machine Learning. Beijing: Tsinghua University Press, 2017. (in Chinese)

[25] Li H, Qi L J, Zhang J H, Ji R H. Recognition of weed during cotton emergence based on principal component analysis and support vector machine. Transactions of the Chinese Society for Agricultural Machinery, 2012; 43(9): 184-189, 196. (in Chinese)

[26] Li J X, Jiang S P. Adaptive threshold image denoising algorithm based on principal component analysis. Infrared Technology, 2014; 36(4): 311-314, 319. (in Chinese)

[27] Wu W H, Tao H M, Xiao S Z, Tang P W. Optimization and implementation of texture feature extraction algorithm for gray level co-occurrence matrix. Digital Technology and Application, 2015(6): 124-126. (in Chinese) doi: 10.19695/j.cnki.cn12-1369.2015.06.090.

[28] Ji J S. Feature selection and its stability for typical geo-objectsof high-resolution remote sensing image. Shanghai: Shanghai Jiaotong University, 2015. (in Chinese)

[29] Han W T, Sun Y, Xu T F, Chen X W, Su K O. Detecting maize leaf water status by using digital RGB images. Agricultural Engineering Technology, 2016; 36(12): 75. (in Chinese)

[30] Hou Q Q, Wang F, Yan L. Extraction of color image texture feature based on gray-level co-occurrence matrix. Remote Sensing for Land \& Resources, 2013; 25(4): 26-32. (in Chinese)

[31] Jiang Y J, Wang X D, Wang W J, Bi K. New feature selection approach by PCA and Relief F. Computer Engineering and Applications, 2010; 46(26): 170-172. (in Chinese)

[32] Nobuyuki O. A threshold selection method from gray-level histograms. IEEE Transactions on Systems, Man and Cybernetics, 1979; 1(1): 62-66. (in Chinese)

[33] Sun B F, Zhao H, Chen L C, Shu S F, Ye C, Li Y D. Identification of ecosystems based on vegetation indices selection algorithm and decision tree. Transactions of the Chinese Society for Agricultural Machinery, 2019; 50(6): 194-200. (in Chinese)

[34] Huang X J, Zhang L. Modified multi-class support vector machine recursive feature elimination for cancer multi-classification. Journal of Computer Applications, 2015; 35(10): 2798-2802. (in Chinese) 